\section{Propin/Propadien-Umlagerung an Aktivkohle}

Von J. F. Cordes und H. GünZler

Hauptlaboratorium und Ammoniaklaboratorium der Badischen Anilin- und Soda-Fabrik A.G., Ludwigshafen am Rhein

(Z. Naturforschg. 15 b, 682 [1960] ; eingegangen am 15. August 1960)

Kürzlich konnten wir über das Isomerisierungs-Gleichgewicht für die beiden $\mathrm{C}_{3} \mathrm{H}_{4}$-Kohlenwasserstoffe Propin und Propadien ${ }^{1}$

$$
\mathrm{H}_{3} \mathrm{C}-\mathrm{C} \equiv \mathrm{CH} \rightleftarrows \mathrm{H}_{2} \mathrm{C}=\mathrm{C}=\mathrm{CH}_{2}
$$

im Temperaturbereich von 100 bis $350^{\circ} \mathrm{C}$ berichten. Die nur in Gegenwart von Katalysatoren - nicht rein thermisch - ablaufende Umlagerung wurde mit verschiede-

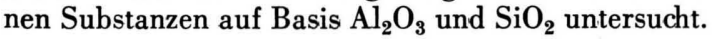

Inzwischen konnten wir feststellen, daß Aktivkohle alle bisher bekannten Katalysatoren im Hinblick auf die Umlagerung in der Gasphase an Wirksamkeit weit über-

1 J. F. Cordes u. H. Günzler, Chem. Ber. 92, 1055 [1959].

2 BASF (Erf.: J. F. Cordes U. H. Günzler), DBP-Anmeldg. B 53532 IV b/12 o. trifft ${ }^{2}$. Werden die reinen Gase bei Temperaturen von etwa 250 bis $300{ }^{\circ} \mathrm{C}$ mit Aktivkohle in Berührung gebracht, so findet innerhalb von wenigen Sek. die Umwandlung in die fehlende Komponente bis zur Gleichgewichtskonzentration statt. Die Geschwindigkeit keiner anderen (Neben-) Reaktion ist in diesem Temperaturbereich mit derjenigen der Isomerisierung zu vergleichen. Im Endgas sind weder ir-spektroskopisch noch gaschromatographisch Produkte von Nebenreaktionen zu erkennen. In Übereinstimmung mit den früher gewonnenen Ergebnissen an den anderen Katalysatoren geringerer Aktivität stellten sich bei $300{ }^{\circ} \mathrm{C}$ stets PropadienKonzentrationen von etwa $19 \%$ ein. Auch die früher gemachten Bemerkungen zur Wirksamkeit der Katalysatoren gelten hier sinngemäß.

Damit lassen sich Propadien und Propin relativ einfach sowie praktisch ohne Verluste präparativ ineinander umwandeln. Die Trennung der anfallenden binären $\mathrm{Ge}$ mische durch Destillation bei tiefen Temperaturen oder unter leichtem Überdruck ${ }^{3}$ bei Zimmertemperatur bereitet dann keine grundsätzlichen Schwierigkeiten mehr.

3 Zum Beispiel wird die Propadien-Propen-Trennung unter Druck von A. Kempkes, Dissertation, Aachen 1959, S. 75, beschrieben.

\section{$\sigma$-Allyl-Manganese Pentacarbonyl and $\pi$-Allyl-Manganese Tetracarbonyl}

\author{
By H. D. Kaesz, R. B. King and F. G. A. Stone \\ Mallinckrodt Chemical Laboratory, Harvard University, \\ Cambridge, Massachusetts, U.S.A. \\ (Z. Naturforschg. 15 b, 682-683 [1960] ; eingegangen am 19. Juli 1960)
}

Transition metal complexes are known in which two, four, five, six, seven or eight carbon atoms are bonded to metals, e. g., $\mathrm{K}\left[\mathrm{C}_{2} \mathrm{H}_{4} \mathrm{PtCl}_{3}\right]^{1}$, $\left[\left(\mathrm{Me}_{4} \mathrm{C}_{4}\right) \mathrm{NiCl}_{2}\right]_{2}{ }^{2},\left(\mathrm{C}_{5} \mathrm{H}_{5}\right)_{2} \mathrm{Fe}^{3}$, $\left(\mathrm{C}_{6} \mathrm{H}_{6}\right)_{2} \mathrm{Cr}^{4}, \mathrm{C}_{7} \mathrm{H}_{7} \mathrm{VC}_{5} \mathrm{H}_{5}{ }^{5}$, and $\mathrm{C}_{8} \mathrm{H}_{8} \mathrm{Fe}(\mathrm{CO})_{3}{ }^{6}$. However, as ligands to metals $3 \pi$-electrons systems have been little investigated. The allyl group might be expected to behave as a formal $3 \pi$-electron donor, just as butadiene can formally be regarded as a $4 \pi$-electron donor in butadiene-iron tricarbonyl ${ }^{7}$. The recent report ${ }^{8}$ of a compound $\mathrm{C}_{3} \mathrm{H}_{5} \mathrm{Co}(\mathrm{CO})_{3}$ which may be regarded as $\pi$-allyl-cobalt tricarbonyl, isoelectronic with butadieneiron tricarbonyl ${ }^{7}$, prompts us to describe a compound which may be regarded as a $\pi$-allyl-manganese complex.

Manganese carbonyl (5.0 g., 12.5 mmoles) in tetrahydrofuran was treated under nitrogen with $7 \mathrm{ml}$. of one

1 Zeise, Mag. Pharm. 35, 105 [1830].

2 R. Criegee u. G. Schröder, Liebigs Ann. Chem. 623, 1 [1959].

3 T. J. Kealy u. P. L. Pauson, Nature [London] 168, 1039 [1951]; S. A. Miller, J. A. Tebboth u. J. F. Tremaine, J. chem. Soc. 1952, 632; G. Wilkinson, M. Rosenblum, M. C. Whiting u. R. B. Woodward, J. Amer. chem. Soc. 74, 2125 [1952] ; E. O. Fischer u. W. P PAB, Z. Naturforschg. 7 b, 377 [1952]. percent sodium amalgam, and stirred at $25^{\circ}$ for $12 \mathrm{hrs}$. to obtain sodium pentacarbonyl manganate (-I) solution. Excess of amalgam was siphoned off, and the solution treated twice with mercury to remove unreacted sodium. To the remaining solution allyl chloride $(7.5 \mathrm{~g}$., $100 \mathrm{mmoles}$ ) was added, and the mixture was stirred at $25^{\circ}$ for three hrs. Solvent was removed $\left(20^{\circ} / 15 \mathrm{~mm}\right.$.) and the residue extracted with three $20 \mathrm{ml}$. portions of n-pentane. After removal of pentane $\left(20^{\circ} / 15 \mathrm{~mm}\right.$.) the oily liquid remaining was transferred $\left(25^{\circ} / 10^{-3} \mathrm{~mm}\right.$.) to a vacuum system, affording 4.9 grams ( $82 \%$ yield) of air-sensitive $\sigma$-allyl-manganese pentacarbonyl, a lemonyellow liquid (Found: C, 40.9; H, 2.4; Mn, 23.8. $\mathrm{C}_{8} \mathrm{H}_{5} \mathrm{O}_{5} \mathrm{Mn}$ requires $\mathrm{C}, 40.7 ; \mathrm{H}, 2.1 ; \mathrm{Mn}, 23,3 \%$ ). The infrared spectrum (liquid film 0.015 mm., Perkin-Elmer Model 21 spectrophotometer, sodium chloride optics) showed a strong sharp band at $1617 \mathrm{~cm}^{-1}$ due to the carbon-carbon double bond stretch of the $\sigma$-bonded allyl group. The carbonyl region of the spectrum (calcium fluoride optics, tetrachloroethylene solvent) showed bands at 1926 (vw), 1993 (s), 2014 (vs), 2046 (vw) and $2108(\mathrm{~m}) \mathrm{cm}^{-1}$. The n.m.r. spectrum of $\sigma$-allylmanganese pentacarbonyl $\left(\mathrm{CS}_{2}\right.$ solution, 40 and 60

4 E. O. Fischer u. W. HAFNer, Z. Naturforschg. 10 b, 665 [1955].

5 R. B. King u. F. G. A. Stone, J. Amer. chem. Soc. 81, 5263 [1959].

6 T. A. Manuel u. F. G. A. Stone, J. Amer. chem. Soc. 82 , 366 [1960].

7 B. F. Hallam u. P. L. Pauson, J. chem. Soc. 1958, 642.

8 R. F. Heck u. D. S. Breslow, J. Amer. chem. Soc. 82, 750 [1960]. 